\title{
De l'image au texte et du texte à l'image : sur les puissances de la peinture chez Boccace
}

\section{Philippe Guérin}

\section{OpenEdition}

\section{Journals}

Édition électronique

URL : http://journals.openedition.org/cei/863

DOI : $10.4000 /$ cei.863

ISSN : 2260-779X

\section{Éditeur}

UGA Éditions/Université Grenoble Alpes

\section{Édition imprimée}

Date de publication : 15 juillet 2008

Pagination : 13-39

ISBN : 978-2-84310-122-9

ISSN : 1770-9571

\section{Référence électronique}

Philippe Guérin, « De l'image au texte et du texte à l'image : sur les puissances de la peinture chez Boccace », Cahiers d'études italiennes [En ligne], 8 | 2008, mis en ligne le 15 janvier 2010, consulté le 19 avril 2019. URL : http://journals.openedition.org/cei/863 ; DOI : 10.4000/cei.863 


\title{
DE L'IMAGE AU TEXTE ET DU TEXTE À L'IMAGE
}

\author{
Sur les puissances de la Peinture Chez Boccace
}

\author{
Philippe Guérin \\ Université Rennes 2
}

\begin{abstract}
C'est une idée de peintre, ou de quelqu'un qui a vu de la peinture; pas forcément le tableau. Mais allons plus loin. Ce ne sont pas des descriptions. C'est une interprétation de...; une herméneutique de... - C'est-à-dire, demanda l'Autre? - C'est-à-dire que l'interprétation établit une distance qui rend vraisemblable (et je n'ai pas dit vraie) la présence du tableau désigné par le geste. «Regarde la peinture». Il faut cette fiction de la réalité, qui est au sophiste comme le fond du tableau au peintre. Mais la réalité objective m'importe assez peu. Je vous rappelle la théorie de la phantasia, la force des apparitions, l'énergie des images, leur évidence. La peinture est là. C'est Philostrate qui peint, avec sa technique de rhéteur ou de poète, comme on voudra.
\end{abstract}

Jackie Pigeaud, Les Loges de Philostrate ${ }^{1}$

Une familiarité même limitée avec l'œuvre de Boccace suffit pour se convaincre rapidement que la conscience chez lui des puissances de l'image est de bout en bout une donnée massive et constante, et qu'une telle conscience s'accompagne d'une science consommée pour solliciter ces puissances, les mettre en branle sous des modalités extrêmement variées. Omniprésentes, les images boccaciennes permettraient même, me semble-t-il, de reparcourir dans son intégralité - ou à peu près - la riche phénoménologie médiévale les impliquant, jusques et y compris sous les espèces de la vision ou du rêve, ou encore sous l'angle de leur pouvoir magique, apotropaïque, voire miraculeux ${ }^{2}$.

1. Jackie Pigeaud, Les Loges de Philostrate, Nantes, Le Passeur/ Cecofop, 2003, p. 58.

2. Je pense ici aux effets de l'adoration des images dans le Filocolo, et tout particulièrement au rôle joué dans la conversion du protagoniste par l'«effigie» du Christ, les «imaginations» qu'elle suscite et le serment qui la prend à témoin (V 52, 53, 54, 66). Voir encore (ibid., IV, 1-3 et V, 37), dans la proximité topique d'une fontaine, le pouvoir qu'a l'image reflétée de Biancifiore de dissoudre le sortilège qui tient Fileno captif. Mais aussi, dans le Décaméron, l'ex-voto de VII 3, 37 et 41 (la "statua» ou "imagine di cera», placée "dinanzi alla figura di santo Ambruogio »; ou encore l'« imagine di stagno » sur quoi repose tout le subterfuge conçu par l'écolier de VIII 7 pour se venger de la veuve qui l’a bafoué (\$56-65). 
Mais ce n'est évidemment pas à un tour d'horizon, même très sommaire, de cette prolifération multidirectionnelle que je vais procéder ici. Ce sont quelques liens intimes de la "virtù fantastica» avec la textualité que je voudrais envisager, quand la "ferma imaginativa" s'y déploie en mobilisant à sa guise la faculté qui est sienne de modeler par l'écriture, les "imaginazioni ${ }^{3}$ ». À vrai dire, la littérature critique concernant les formes et les enjeux - du dialogue entre texte et image chez Boccace est déjà fort abondante - qu'il suffise de citer pour mémoire le dernier grand-œuvre coordonné par Vittore Branca, ou bien encore le beau Ragionare nel giardino de Lucia Battaglia Riccí ${ }^{4}$. Mais c'est un point de vue plus interne que je voudrais adopter ici: en effet, les choses sont envisagées assez généralement sous l'angle de l'image comme motif dans le texte ${ }^{5}$ ou de l'œuvre comme prétexte à images qui en découlent, ou encore d'un dialogue qui se noue au plan des contenus et des messages, des signifiés véhiculés par des systèmes sémiotiques différents - sans que le procès de signification en tant que tel, susceptible pourtant d'affecter l'une et l'autre des parties de cet "échange», soit à mon sens suffisamment examiné. Pourtant, il est souvent des allusions dans le texte même de Boccace à ces passages transsémiotiques ${ }^{6}$, où l'écriture non seulement se nourrit d'images censées lui préexister, mais est dotée aussi d'une vis imaginativa propre, consubstantielle à la production même du texte et, partant, à ses effets.

Pour tenter de mieux cerner la question, faisons un détour par la postérité. Commentant l'ode Puella de Politien, Mario Martelli fait remarquer que,

3. Cf. Corbaccio, 27; Elegia di Madonna Fiammetta, VI 10, 9; Comedia delle ninfe fiorentine, XXVIII, 9.

4. Boccaccio visualizzato. Narrare per parole e per immagini fra Medioevo e Rinascimento, éd. V. Branca, 3 vol., Turin, Einaudi, 1999; Lucia BATTAGLIA RICCI, Ragionare nel giardino. Boccaccio e i cicli pittorici del Trionfo della Morte, Rome, Salerno Editrice, $2000^{2}$.

5. Cf. Creighton Gilbert, «La devozione di Giovanni Boccaccio per gli artisti e l'arte», in Boccaccio visualizzato, cit., I, p. 145-153 (l'auteur de cette étude a le mérite de renverser - c'est ce que dans son introduction il ambitionne explicitement de faire - la perspective habituelle, celle qui va du texte aux images, extérieures à lui, qui en sont issues). Il arrive aussi que dans le texte boccacien l'image peinte soit virtuelle: c'est le cas de la description de la figure allégorique de la Courtoisie dans Décaméron I 8 (mais c'est alors l'image comme productrice de sens qui est en tant que telle thématisée: ce point mériterait aussi quelques développements).

6. Cf. par exemple Filocolo, III 23, 3 (la missive de Biancifiore lui ayant été remise, «Florio aperse la ricevuta lettera, e quella infinite volte rilesse pensando alle parole di Biancifiore, sopra le quali faccendo diverse imaginazioni, sopra il suo letto con essa lungamente dimorò »).

7. Cf. Mario Martelli, Angelo Poliziano. Storia e metastoria, Lecce, Conte, 1995, p. 280-301. On peut lire l'oda In puellam suam avec traduction italienne en regard dans Poeti latini del Quattrocento, éd. F. Arnaldi, L. Gualdo Rosa, L. Monti Sabia, Milan-Naples, Ricciardi, 1964, 
si les douze premiers vers de ce petit chef-d'œuvre de l'expression lyrique latine du Quattrocento sont le produit d'un assemblage raffiné de sources classiques, puisées au trésor de la science infinie de l'auteur en la matière, à partir du vers 13, en revanche, les modèles lui sont fournis par la tradition médiévale de la descriptio mulieris - toute contaminée qu'elle reste, dans le travail de marqueterie d'Agnolo Ambrogini, de réminiscences antiques ${ }^{8}$. Une place de choix, dans ce cheminement à rebours, revient à Boccace, à côté (ce ne sont que quelques exemples) du Detto d'amore (v. 167-225), du Mare amoroso (v. 90-100 et 120-135), du Tesoretto (v. 248-271): Boccace, c'est-à-dire essentiellement, en l'occurrence, l'auteur des trois chapitres de la Comedia delle ninfe forentine consacrés à l'évocation de la nymphe Lia et de ses compagnes. La série des six portraits constitue sans conteste, aux yeux de Martelli, «il caso più impressionante di applicazione di questa formula [i.e. de la descriptio extrinseca $]^{9}$ ".

Mais Martelli, qui, pour les antécédents proches (en gros les deux siècles qui précèdent l'âge laurentien), se limite presque exclusivement à la production italienne en langue vulgaire, ne cite pas le texte qui pourrait bien jouer un rôle décisif au point de départ de la récupération d'une telle tradition par Boccace ${ }^{10}$. Giuseppe Velli, éditeur scientifique et commentateur des Carmina, et donc du tout premier essai de jeunesse, connu comme Elegia di Costanza, indique en effet que le portrait de la défunte par son amant éploré dénonce de façon manifeste la lecture de l'Anticlaudianus d'Alain de Lille ${ }^{11}$. Et plus précisément des vers 270-302 du livre I, qui correspondent à la description physique du personnage de Prudencia, au moment de son entrée en scène dans la vaste construction

p. 1050-1059; elle figure aussi dans la récente Anthologie de la poésie lyrique latine de la Renaissance, édition bilingue de P. Laurens, Paris, Gallimard, 2004, aux p. 78-87.

8. À ces sources médiévales, il convient d'ajouter les Écritures, filtrées ou non (et tout particulièrement le Cantique des cantiques d'où descend le filon dont nous entendons nous occuper ici).

9. Cf. M. MarTelli, Angelo Poliziano, cit., p. 283.

10. Ce texte n'est pas cité non plus (mais pour des raisons plus compréhensibles, tenant à la culture de l'auteur qu'il étudie, de nature bien différente) par Paolo ORVIETO - nous sommes toujours au Quattrocento, tout près chronologiquement et spatialement de Politien - dans son analyse de la "descriptio Anteae" du Morgante de Luigi Pulci (Pulci medievale. Studio sulla poesia volgare fiorentina del Quattrocento, Rome, Salerno Editrice, 1978, p. 48-85); le rôle charnière de Boccace est fortement souligné ici aussi (notamment: «sarà Boccaccio ad attuare la definitiva retorizzazione e laicizzazione del canone», p. 58).

11. Cf. Tutte le opere di Giovanni Boccaccio, éd. V. Branca, V, 1: Rime. Carmina. Epistole e lettere. Vite. De Canaria, Milan, Mondadori, 1992, p. 404-411; voir en outre l'introduction, ibid., p. 378-379 et, pour la note qui concerne le présent propos, p. 468. Voir aussi, du même G. VeLLI, in Petrarca e Boccaccio. Tradizione, Memoria, Scrittura, Padova, Antenore, 1979, les deux chapitres - le premier, surtout - intitulés "Sull'Elegia di Costanza» et "L'Elegia di Costanza e l'ars combinatoria' del Boccaccio ", respectivement aux p. 97-111 et 112-121. 
allégorique du poème commis par le prieur bénédictin de Canterbury ${ }^{12}$. Je me propose donc d'essayer de dégager quelques lignes de force quant aux enjeux de ce qui sera chez le Boccace postérieur à l'Elegia un exercice de variatio-amplificatio sans cesse reprise et poussée à la limite (à la limite de la réécriture, y compris et avant tout de soi, en une forme de «maniérisme» ostentatoire), de l'application virtuose sur ce que Pozzi appelait le «canon long» (par opposition au «canon bref» pétrarquien) de la descriptio puella ${ }^{13}$. Après avoir souligné au passage que cette unité discrète d'une descriptio prête au réemploi, disponible comme image-signe pour d'autres usages, c'est précisément chez Alain de Lille que Boccace la trouve d'abord. Autrement dit, chez l'un de ceux qui, pour Edgar De Bruyne déjà, témoignent au plus haut point de la revalorisation des images à partir du XII ${ }^{e}$ siècle - mouvement qui se traduit aussi bien par l'élaboration d'une théologie sur le sujet de plus en plus articulée que par le formidable essor des productions concrètes et les changements profonds qui les caractérisent - ceux-ci précédant souvent celle-là et appelant a posteriori une légitimation en théorie ${ }^{14}$. Nous aurons à revenir sur toutes ces questions.

Avant de regarder de plus près ce qu'il en est dans l'Ameto (ainsi qu'on appelait naguère la Comedia delle ninfe), il convient de remarquer que l'Elegia déjà inverse le sens de l'ekphrasis d'Alain de Lille, traitée (à l'instar des vers sur les "poma mamillarum" repris verbatim) ${ }^{15}$ comme macrosigne pour une opération, plutôt simple ici, de recontextualisation. Celle-

12. Je renvoie à l'éd. suivante: AlANo Di Lilla, Viaggio della saggezza. Anticlaudianus, Discorso sulla sfera intelligibile, a cura di C. Chiurco, testo latino a fronte, Milan, Bompiani, 2004. Le passage cité est aux p. 104-106.

13. Cf. Giovanni PozzI, Sull’orlo del visibile parlare, Milan, Adelphi, 1993, les chapitres 4 («Il ritratto della donna nella poesia d'inizio Cinquecento e la pittura di Giorgione») et 5 ("Nota additiva alla descriptio puellae»), p. 145-184. Il mentionne à propos des descriptions de la Comedia delle ninfe "la serie più spettacolosa di canoni lunghi di tutta la letteratura" (p. 152), et l'Ameto est " l'opera dove l'esercizio su quella variante è spinto fino al parossismo" (p. 177). Plus curieusement encore que dans les cas précédents, Pozzi "oublie» à son tour Alain de Lille, pourtant cité dans d'autres études du volume (mais pas l'Anticlaudianus). Pour une variation sur le "canon bref" due à Politien, v. les Stanze, I 42-44, brièvement mentionnées par MARTELli, op. cit., p. 294, et PozZI, p. 155. Il conviendrait de compléter l'enquête en l'élargissant au De planctu Nature, où la Nature est dépeinte en des termes très proches (et l'on notera que, même si la confrontation des deux textes interdit de penser qu'il puisse en dériver, c'est de Natura qu'il s'agit aussi dans le Tesoretto de Brunetto Latini).

14. Cf. Edgar De Bruyne, Études d'esthétique médiévale, Bruges, De Tempel, 1946, que je cite d'après la réédition Paris, Albin Michel, 1998, 2 t., I, p. 656-671.

15. Cf. les v. 290-291 d'Alain et 72-73 de Boccace. 
ci nous fait passer de la troisième personne de l'invention allégorique à l'artifice d'une énonciation dialogique qui voit le lecteur-passant-amant inclure dans sa "réponse», mais sous les espèces de la "non-personne» benvenistienne, l'évocation de l'absente: la nostalgie poignante y est signifiée par l'image telle que (re)produite dans l'univers sémiotique de l'écriture. C'est toujours un lien avec l'invisible qu'il s'agit d'établir. Mais chez Alain de Lille, l'integumentum ${ }^{16}$ de la pictura - cette ars comme la poésie supérieure à la logique, susceptible de dépasser la nature et ses forces - cet "intégument» (comme dira encore Christine de Pizan, par exemple), qui est certes simia (ou histrio) ueri et donc potentiellement dangereux, est, comme la vision onirique inspirée et à condition d'être employé à bon escient, transitus vers les arcanes divins: de telle sorte que l'appel à l'étreinte que lance l'image de Prudencia est une invitation à chercher sous le portrait (vultus) d'autres réalités que celles qu'elle montre, des réalités cachées et meilleures encore; dans l'ombre des choses qu'elle représente, la peinture fait par ses signes parler les mystères. Telle est la logique de la translatio, fondée sur une "métaphysique de la forme» de matrice boécienne, qui se déploie chez Alain ${ }^{17}$. Chez Boccace, tout au contraire, dans son juvénile exercice d'apprentissage modelé sur une épitaphe latine (sa source première), le voile de l'image recouvre l'atroce réalité de l'absence signifiée par le tombeau, une image qui ravive le désir en son impasse «mélancolique», ranimant par un tel artifice son objet, tout en le posant comme inatteignable. Le travail mémoriel dessine l'espace d'un drame: les bras n'embrasseront jamais, jamais le jeune homme ne verra les parties cachées qu'il pensait plus belles que celles exposées jusque-là à sa vue. L'image s'est faite pierre, la mort n'est que ténèbre.

Notons pour finir sur ce premier excursus qu'entre l'Elegia et le prosimetrum de la Comedia, il est une tentative intermédiaire, mise en chantier, sinon entièrement rédigée, à la toute fin de la période napolitaine, immédiatement antérieure, donc, à ce qui va être examiné sous peu: il s'agit du portrait de l'héroïne du Teseida delle nozze di Emilia (XII, 53-63). Dans cette suite de onze huitains, outre le contexte entièrement profane, la

16. L'integumentum y est même l'objet d'une figuration dans la figuration, puisque les figures allégoriques portent des vêtements "historiés", prétextes à longues ekphraseis.

17. Cf. Anticlaudianus, le Prologus en prose, ainsi que I, 119sq.; II, 204sq.; III, 33; etc. Voir aussi l'introduction de C. Chiurco à l'éd. cit. de l'Anticlaudianus, en particulier les p. 23-25 et 3340 , ainsi qu'ibid. les notes de commentaire aux (très nombreux) passages où intervient la question de la peinture et des images. 
fusion inédite des motifs canoniques, topiques au sens propre (et mâtinés de couleurs stilnovistes) dans un mètre "épique», la description ainsi fortement narrativisée est orientée vers son au-delà: elle vise la continuation fantasmée par le lecteur - mais fortement suggérée par le texte - du récit des noces consommées, quand les charmes cachés de l'épouse n'auront plus de secret pour le regard (les yeux du corps) de l'époux les découvrant émerveillé. Cette tension, préfigurée par des détails à la forte charge érotique, visant à donner force de chair au portrait ${ }^{18}$, fait du langage verbal le vecteur d'images qui vont jusqu'à relever le défi de la tridimensionnalité, et l'incitateur d'autres images, purement mentales cette fois, confiées au jeu de la libre fantaisie du lecteur. Voilà ce que cachent les «dessous de l'image-femme» chez Boccace, ce qu'appelle la part d'invisible qui lui est consubstantielle.

C'est une telle orientation qu'approfondit et porte à son acmé la Comedia delle ninfe fiorentine $e^{19}$. On se rappelle la situation initiale: Ameto, ayant surpris le groupe des nymphes qu'il prend pour des déesses descendues du ciel, manque, tel Actéon, d'être dévoré par leurs chiens, après les avoir contemplées en train d'ouvrir leurs robes, en quête de fraîcheur, pour laisser passer sur leur peau un peu d'air (chapitre III). Puis, après une application du "canon bref» à la description de la rencontre particulière avec Lia au chapitre $V$, les six autres nymphes se présentent deux par deux, aux chapitres numérotés IX, XII et XV - les valeurs numérologiques affichées leur conférant évidemment une fonction stratégique. On a le sentiment d'assister à un jeu de variations subtil et raffiné sur un motif unique. Mais ces variations sont à examiner, je crois, en détail, comme ce qui, au-delà de la récupération en une série d'une rare densité, à prétention totalisante, de toute la littérature antérieure sur le sujet (la sienne comprise), ainsi que de ses justifications théoriques, fait trembler ou vibrer le système, au risque (calculé) de le voir s'effondrer sur luimême. En d'autres termes, se joue déjà ici la dialectique de l'ordre parfait, mesuré et rationnel (species, ordo et modus du canon reçu en héritage) et de ce qui, relevant de la liberté du jeu, l'anime, le rend vivant - cette dialectique qui, sur un autre plan, sera portée au plus haut de ses possibilités

18. Cf. notamment les quatre derniers vers du huitain 61 : «e'l petto poi un pochetto eminente / de' pomi vaghi per mostranza tondi, / che per durezza avean combattimento, /sempre pontando in fuor, col vestimento». La fermeté des seins (la "glose» traduit les "pomi» en termes propres, pour qui n'aurait pas compris), trait constant dans tous les textes examinés jusqu'ici, actualise ici ce qui restait virtuel dans les vers de l'Elegia où Boccace citait littéralement Alanus.

19. Je renvoie à l'édition suivante: Tutte le opere..., cit., II : Filostrato. Teseida della nozze di Emilia. Comedia delle ninfe fiorentine, Milan, Mondadori, 1964. 
avec le récit-cadre du Décaméron. L'extrême préciosité des descriptions est certes au service d'un type idéal et abstrait; conforme en cela à l'«esthétique» médiévale, elle est le miroir du modèle unique et absolu de la beauté féminine. Mais en même temps, le jeu raffiné et «maniériste» de la variatio introduit des tremblements dans l'image, les mouvements quasi imperceptibles de l'une à l'autre qui lui font subir autant d'altérations ténues la mettant en mouvement, l'orientant vers l'individuation - toute relative que celle-ci demeure. $\mathrm{Ou}$, plus exactement, elle oscille entre la répétition du même - la projection selon les topoi les plus éprouvés du désir d'amour d'Ameto - et l'incarnation des formes que prend ce désir unique et mobile dans des objets capables de se renouveler en se singularisant un tant soit peu. Quelques indications rapides pour appuyer le propos: les justes rondeurs de telle ou telle partie imposent de regarder le reste, les reliefs du vêtement dénoncent la ferme poitrine juvénile que nous avons rencontrée dès l'Elegia; dès lors l'imagination («l'occhio mentale») se met en quête de ce qui reste caché ${ }^{20}$. Dans le rigoureux respect de l'ordre descendant requis par le canon, la gorge s'offre à la suite du menton, le décolleté de la robe aimante le regard du berger, attiré à plusieurs reprises sur une voie menant à une demeure céleste, et les «reliefs" sont une invitation faite à la main pour qu'elle se glisse sous le vêtement ${ }^{21}$. Une sueur délicate est prétexte à geste gracieux, trois paragraphes peuvent être consacrés à une chevelure où l'ordre parfait de la coiffure laisse néanmoins échapper quelques mèches en liberté, rendant la nymphe si "cianciosa" que s'éveille l'envie de doux baisers, redoublée par le désir que font naître les bras d'étreintes répétées; chaque ouverture dans le vêtement est prétexte à coups d'œil furtifs, et l'appétit aussitôt de s'enflammer ${ }^{22}$. Les yeux larrons et la bouche "vermigliuzza" (relevons aussi le nombre des diminutifs et leur qualité) sont de nouvelles invites aux baisers, et le fruit de ce

20. Comedia, IX, 16-20. Pour «l'occhio mentale» qui passe au-delà de l'étoffe des habits, v. XII, 28.

21. Ibid., IX, 25-27. On peut rapprocher ce passage de celui du Filocolo (IV, 118, 6) où la main, guidée par l'œil, se faisait beaucoup plus audacieuse (mais dans une situation bien différente: les deux amants se retrouvent pour la première fois dans le même lit) : «Egli la scuopre [du drap qui la couvre] e con amoroso occhio rimira il dilicato petto, e con disiderosa mano tocca le ritonde menne, baciandole molte volte. Egli distende le mani per le segrete parti, le quali mai amore ne' semplici anni gli avea fatte conoscere, e toccando perviene infino a quel luogo ove ogni dolcezza si richiude: e così toccando le dilicate parti, tanto diletto prende, che gli pare trapassare di letizia le regioni degl'iddii ».

22. Ibid., XII, 7-9, 11-12, 14, 16; "cianciosa" (pour "gracieuse», $\$ 7$ ) est un napolitanisme d'autant plus savoureux que ce sont des nymphes florentines, cette fois. Pour les mèches, v. aussi $\mathrm{XV}, 24$. 
qu'a vu l'œil se trouve dans les recoins dérobés à la vue ${ }^{23}$. S'y ajoutent des détails tout à fait exceptionnels, si je ne m'abuse, dans l'avant-dernier tableau: les oreilles, dont se sert artistement la coiffure, entrent dans le champ de vision, de même qu'une jambe ronde et sans bas ${ }^{24}$; prolongent les bras des mains par lesquelles on voudrait être serré et touché; le vêtement s'ouvre latéralement sur les flancs, l'appétit connaît un dernier sursaut... ${ }^{25}$. Ameto n'a évité de perdre la tête que parce qu'il a estimé qu'il était au paradis ${ }^{26}$.

Sur le plan quantitatif, on notera pour finir que le nombre de lignes consacrées à ces descriptions va croissant jusqu'à la quatrième, pour décroître très rapidement dans la dernière - la plus courte de la série ${ }^{27}$. Essoufflement? Peut-être, mais si l'on rassemble les détails "érotiques» pour les lire en une suite de variations sur les figures du désir, la série se propose comme le mime d'une étreinte amoureuse fantasmée, avec ses longs préliminaires, son acmé et la chute rapide qui s'ensuit - même si l'œil jusqu'au bout reste "luxurieux ${ }^{28}$ ». Voilà sur quoi débouche l' "addensamento iconico-descrittivo" dont parle Marcello Ciccuto dans un autre contexte ${ }^{29}$. Le dernier passage en revue du chapitre XXVIII le dit on ne peut mieux $^{30}$.

\section{Ibid., XII, 23, 25, 28, 30.}

24. Sur les parties constitutives du canon pétrarquien (dont, comme le nez, sont absentes les oreilles) et sa descendance, v. bien sûr Amedeo QuONDAM, Il naso di Laura. Lingua e poesia lirica nella tradizione del Classicismo, Modène, Panini, 1991, p. 291-328.

25. Comedia, XV, 13, 18-19, 23, 25.

26. Ibid., X, 5-6; XV, 15.

27. Voici le nombre de lignes qu'occupe chacun des portraits dans l'édition de référence: 1 $=40 ; 2=44 ; 3=62 ; 4=76 ; 5=60 ; 6=36$.

28. Comedia, XV, 26.

29. "Tra Zibaldoni e Decameron: Boccaccio disegnatore», in Marcello CiCCuTO, Figure d'artista. La nascita delle immagini alle origini della letteratura, Fiesole, Cadmo, 2002, p. 105-123 (étude parue dans une première version sous le titre «Immagini per i testi di Boccaccio : percorsi e affinità dagli Zibaldoni al Decameron" in Gli Zibaldoni di Boccaccio. Memoria, scrittura, riscrittura, Atti del seminario internazionale di Firenze-Certaldo [26-28 aprile 1996], éd. M. Picone/C. CazaléBérard, Florence, Cesati, 1998, p. 141-160).

30. Comedia, XXVIII : les contemplant tour à tour - et la dernière regardée étant toujours la plus belle - Ameto, «mirandole effettuosamente con ardente disio, in se medesimo fa diverse imaginazioni concordevoli a'suoi disii. Egli alcuna volta imagina d'essere stretto dalle braccia dell'una e dell'altra strignere il candido collo, e quasi come se d'alcuna sentisse i dolci baci, cotale gusta la saporita saliva; e tenente alquanto la bocca aperta, nulla altra cosa prende che le vane aure. Poi, più innanzi con la imaginazione procedendo, si pensa dovere ad alcuna scovrire i suoi disii e tremebundo diventa. E già nel pensiero non conosce come essere possa che gliele possa dire, ma pure, parendogli quasi averne sopra la verde erba con parole convertita alcuna, d'allegrezza fatto caldissimo, sé tutto di sudore bagnato dimostra [...]"; et, une nouvelle fois, «la ferma imaginativa di lui vag[a] per le segrete parti di quelle». 
Mais c'est aussi que les nymphes savent fort bien de quoi il retourne: il suffit de mentionner l'artifice auquel recourt Mopsa (au chapitre XVIII) pour vaincre l'insensibilité de celui sur lequel elle a jeté son dévolu. C'est dès lors tout le sens de la construction allégorique de la Comedia qui en est affecté. En effet, ce prosimètre, qui devrait connaître son dénouement dans l'hymne du chapitre XLVII (préparé par les différentes étapes de la purification dans les chapitres précédents), où est enfin révélé le sens du parcours initiatique d'Ameto vers les réalités célestes et la lumière divine, connaît en réalité une conclusion bien ambiguë31 : le narrateur (figure de l'auteur) n'est quant à lui pas sorti de sa propre impasse, il est condamné à choir encore et toujours dans un réel évoqué sous un jour infiniment moins plaisant que celui des bois peuplé de nymphes. Le spectacle auquel il a assisté n'est en fin de compte qu'un rêve par procuration auquel il ne nous demande pas même de croire - une chimère, moins qu'une vision. On a insisté à juste titre - en y voyant un terrain d'expérimentation pour le futur auteur du Décaméron - sur le goût de la narration qui se manifeste dans les récits autobiographiques (centrés sur leurs amours) auxquels se livrent tour à tour les nymphes. Boccace cherche quelque chose d'analogue dans le perfectionnement maniériste des potentialités de l'image, en un jeu virtuose sur leur réversibilité - leur ambiguïté, tout comme est ambigu l'édifice entier de la Comedia en tant que vaste image allégorique. Comment prendre tout à fait au sérieux le narrateur du chapitre XLVI, qui nous dit que dorénavant les nymphes plaisent davantage à l'intellect d'Ameto qu'à ses yeux, quand d'abord c'était l'inverse? Le balancement en tout cas semble ne devoir connaitre que difficilement son terme.

Il suffira à Politien de changer de régime énonciatif, en sa longue adresse à la puella, apostrophe tout entière vouée à évoquer un passé récent et encore vivant dans la chair de l'amant éconduit, pour actualiser les virtualités de l'ekphrasis, en adjoignant au catalogue canonique reçu en héritage quelques "parties» inédites et en passant continûment de la vision mémorielle au souvenir du toucher ${ }^{32}$. Exercice humaniste, certes, mais dont la

31. Une telle ambiguiité a été mainte fois relevée: voir par exemple Carlo MusceTtA, Boccaccio, Rome-Bari, Laterza (LIL), $1980^{3}$ [1972], p. 99-110; Luigi SuRDICH, Boccaccio, Rome-Bari, Laterza, 2001, p. 58-67.

32. Le souvenir de la bouche évoque les lèvres mordillées (v. 43-46), les langues et les haleines s'entremêlant, hâtant ainsi l'orgasme (v. 49-56); celui des seins, les baisers et les caresses (v. 6268); entre la poitrine et les pieds mignons, le ventre, les cuisses, les hanches de la "puella" viennent compléter le portrait. 
fascination érotique qu'il dégage ne pâtit nullement de ce qu'il est imitation: car, outre les détails visuels faits pour solliciter l'imagination, il introduit dans l'image de façon beaucoup plus prégnante encore la dimension du temps - ce qui est le propre de l'image verbale et de sa capacité de suggestion. Mais, pour faire retour vers les images proprement visuelles et ce qu'elles doivent au legs boccacien, il faudrait aussi donner corps - si j'ose dire - à la suggestion de Pozzi : après avoir examiné trois cas de traduction picturale du "canon bref» de la poésie (en particulier la «Laure» de Giorgione), celui-ci prolonge le propos - en restant toutefois allusif: il ne s'arrête un instant que sur la Vénus de Dresde du Titien - sur le «canon long» de la prose, celui qui fait de Boccace le maillon essentiel, la charnière de la longue chaîne de la descriptio mulieris, depuis les dames des vergers de Déduit jusqu'au nu moderne. Et aux exemples qu'il cite, les Vénus, Madeleine, Suzanne, etc., il faudrait sans doute - outre Eve bien sûr - ajouter Daphné: dans l'Ameto, les nymphes sont (à l'ombre des lauriers) les égales en beauté de celle qu'aima Phébus et cette dernière apparaît alors comme le terme de comparaison adéquat pour évoquer la force du désir d'Ameto - Boccace ne se faisant pas faute d'indiquer que Daphné eût certainement changé d'avis et préféré l'étreinte d'Apollon à la métamorphose, si elle avait su à temps à qui elle avait à faire ${ }^{33}$.

Mais c'est aussi que les nymphes se prêtent mieux que toute autre figure aux jeux de l'imagination - dans toutes les acceptions possibles (médiévales) du terme. Je rappellerai brièvement ici un aspect de la thèse développée par Giorgio Agamben dans un article récent, qui prolonge sur ce point les recherches entamées jadis par Aby Warburg ${ }^{34}$ : les nymphes sont des "cristaux de mémoire historique», chez qui «l’originaire et la répétition, la forme et la matière se font indiscernables»; de l'ordre des «fantasmata" (et des Pathosformeln warburgiennes), elles ont la faculté de se charger de temps, elles combinent "création et performance», "singularité inaugurale et répétition ${ }^{35}$ ». Elles mêlent, entre conscient et inconscient, la liberté (du vivant) et la contrainte (du «déjà-là», de l'image inanimée) en un jeu qui est «oscillation polaire»; c'est en «dynamogrammes» que

33. Cf. Comedia, respectivement XXXI, 14; XII, 16; XVIII, 20.

34. Il s'agit de "Nymphae», article inédit de 2003 publié comme second chapitre de Giorgio Agamben, Image et mémoire. Ecrits sur l'image, la danse et le cinéma, traduit par M. Dell'Omodarme, S. Doppelt, D. Loayza et G.A. Tiberghien, Paris, Desclée de Brouwer, 2004, p. 37-69.

35. Ibid., p. 43-44. 
s'actualise leur "potentiel cinétique» d'images au "destin spectral». Toutefois, dans ce procès d'actualisation, les nymphes boccaciennes déboucheraient - selon Agamben - sur une scission de l'imago médiévale, empêchée par une polarisation désormais exacerbée de se résorber en une "vie unifiée» - la scission d'où naîtrait précisément la littérature moderne ${ }^{36}$. Cette vie unifiée dont le philosophe italien s'était mis en quête dans les chapitres de Stanze où il tentait de faire le point sur la "pneumofantasmologie» médiévale ${ }^{37}$. Dans la spéculation de miroir en miroir en quoi consiste tout processus cognitif ${ }^{38}$, le spiritus phantasticus, par le biais duquel se trouve dépassée toute opposition irréductible entre l'âme et le corps, est l'agent de la jonction entre corporel et incorporel, le lieu de la rencontre du rationnel et de l'irrationnel, de l'humain et du divin. En lui réside la possibilité même de l'intellection comme de la signification. C'est cette fonction essentielle de l'image et, partant, de l'imagination que toutes les études sur la question mettent immanquablement en exergue ${ }^{39}$. Mais en réalité, en exacerbant la polarisation de l'image entre canon et individuation pour d'autres fins que la jonction de l'immanent et du transcendant, Boccace la redynamise, lui imprimant de la sorte, comme nous le verrons mieux encore dans les lignes qui vont suivre, une fonction et une direction nouvelles.

Pour ce qui est de l'amour, celui-ci - ainsi que l'enseignent les figures de Narcisse et de Pygmalion - est toujours et d'abord amour d'une image ${ }^{40}$,

36. Ibid., p. 47-49, 54, 64 .

37. Cf. Giorgio Agamben, Stanze. La parola e il fantasma nella cultura occidentale, Turin, Einaudi, 1977, nouvelle éd. 1992. Je cite d'après l'édition française, Stanze. Parole et fantasme dans la culture occidentale, traduit de l'italien par Y. Hersant, Paris, Payot \& Rivages (pour la nouvelle éd.), 1994, 1998 (éd. poche).

38. Cf. ibid., p. 136. En ce qui concerne cette fonction du miroir, qui permet de voir ce que la vue ne pourrait soutenir, pour en rester à un exemple puisé chez Alain de Lille, et dans le sillage de la réflexion sur l'«écriture en images», v. le livre VI de l'Anticlaudianus.

39. Voir par exemple, pour une introduction à cette problématique complexe, outre E. De BRUYNE, Études..., cit., en particulier I, p. 588sq. (sur les "conditions psychologiques» de " l'esthétique des Victorins»), Jean-Claude SCHMITT, «Imago: de l'image à l'imaginaire», et Jean WIRTH, "Structure et fonction de l'image chez saint Thomas d'Aquin", in L'Image. Fonctions et usages des images dans l'Occident médiéval, Actes du $\sigma^{\mathrm{e}}$ "International Workshop on Medieval Societies» (17-23 octobre 1992), sous la direction de J. Baschet et J.-C. Schmitt, Paris, Le Léopard d'or, 1996, respectivement aux p. 29-37 et 39-57; ainsi que (pour un dense panorama sur la longue durée de l'art religieux en Occident) Hans Belting, Bild und Kult. Eine Geschichte des Bildes vor dem Zeitalter der Kunst, Munich, Beck, 1990 (éd. française Image et culte. Une histoire de l'art avant l'époque de l'art, Paris, Cerf, 1998), Jean WIRTH, L'Image médiévale. Naissance et développements (VI'-XVe siècle), Paris, Klincksieck, 1989 et Jean-Claude SCHMITT, Le Corps des images. Essais sur la culture visuelle au Moyen Âge, Paris, Gallimard, 2002, notamment p. 21-31 et 345sq. 40. Cf. G. Agamben, Stanze, cit., p. 137. 
et son pouvoir virtuellement sidérant peut conduire à l'amor hereos, qui l'apparente à la mélancolie, quand "le désir entraîne imagination et mémoire [inextricablement solidaires l'une de l'autre à l'intérieur d'un tel cadre théorique] dans une ronde obsédante [telle la ronde des nymphes, justement: aussi bien dans les représentations où elles sont surprises au bord de l'eau qui les réfléchit que dans le mouvement itératif de leur description deux par deux] autour du fantasme qui s'imprime en elles toujours plus fortement, selon un cercle tellement vicieux, qu'Eros finit par se couvrir du masque saturnien de la pathologie mélancolique ${ }^{41}$ ». Je reviendrai un peu plus loin sur l'attitude d'Ameto au début du chapitre $\mathrm{V}$ de la Comedia. Mais on pressent que pourrait se jouer autre chose aussi dans l'image boccacienne, telle que l'écriture la retravaille: tout se passe en effet comme si l'image verbale pour son propre compte, déployant sa logique spécifique, était mue chez Boccace par la tentation de l'excès par rapport à sa fonction première, par une provocation de sa matérialité sensible face à l'abstraction dont elle est le signe ${ }^{42}$. Aux dépens du "graphisme» strictement codifié de la représentation - là où, autour de ce qu'ils partagent, dessin et écriture trouvent leur point de jonction. En d'autres termes, on retrouverait dans le domaine des études philologiques senso latu la problématique qui a conduit depuis deux ou trois décennies l'histoire de l'art à la critique de l'iconologie ${ }^{43}$.

Car avec la Comedia delle ninfe, qui "déborde» les représentations antérieures, tant visuelles que verbales, et ébranle le système, l'image et ses pouvoirs n'ont pas dit leur dernier mot. La voie explorée là va finir en effet par constituer aux yeux de Boccace une (relative) impasse, au moins pour ce qui est de sa production en langue vulgaire; et c'en est une autre, autrement féconde sans doute, qu'il va par la suite emprunter; mais ce ne sera plus celle de l'ekphrasis. De ce point de vue, l'Amorosa visione, qui met en scène les puissances rhétoriques de la peinture nouvelle ${ }^{44}$, exhibe les

41. Ibid., p. 189.

42. Cf. Georges Didi-Huberman, «Imitation, représentation, fonction. Remarques sur un mythe épistémologique», in L'Image. Fonctions et usages..., cit., p. 59-86, ici p. 83-85.

43. Voir par exemple J. WirTh, L'Image médiévale, cit. (pour la promotion d'une «logique» propre de l'image, constituant l'armature théorique des analyses conduites ensuite dans l'ouvrage, v. le cadre proposé en introduction, p. 7-45); Georges Didi-Huberman, Devant l'image. Question posée aux fins d'une histoire de l'art, Paris, Minuit, 1990; Daniel ARASSE, On n'y voit rien. Descriptions, Paris, Denoël, 2000.

44. On se souvient des vers toujours cités prononcés par le narrateur (personnage-poète, pour la première fois chez Boccace) lorsqu'il pénètre dans le "noble château» et découvre la salle peinte (IV, 13-18) : «Humana man non credo che sospinta / mai fosse a tanto ingegno quanto in quella / mostrava [rédaction B: "mostrante"] ogni figura lì distinta, / eccetto se da Giotto, al qual la bella / 
conditions de son propre dépassement. Certes, on y retrouve, sous les espèces de la «donna gentile» qui flanque Amour sur la quatrième paroi, un portrait susceptible de trouver sa place dans la série dessinée précédemment (le senhal «fiammetta» en l'occurrence nous indique clairement le référent); mais, quoique le genre poétique soit ici encore narratif, c'est au "canon bref", à la fois distendu et ramené à l'essentiel (pour le nombre de ses éléments), que Boccace recourt (les yeux, le front, la bouche; les roses et, dans la deuxième rédaction, les perles); d'autre part, l'image tend tellement à sortir d'elle-même, à faire oublier dans et par les mots sa fixité synchronique d'image peinte, que non seulement la scène s'anime de personnages secondaires saisis en mouvement ou que les figures y sont douées de la faculté de parler, mais, éveillant d'autres sens que la vue seule, elle dégage une odeur, semblable à celle de la panthère ${ }^{45}$. Et l'on retrouve, dans le passage en revue des diverses amours dont la description occupe les chants suivants tel ou tel détail évocateur, et pour maint d'entre eux désormais familier ${ }^{46}$. Pour ne pas parler enfin de l'épilogue une nouvelle fois éminemment ambigu, de sa dimension in fine exclusivement mondaine malgré la loi du genre - ainsi qu'il a été mainte et mainte fois souligné... Mais surtout, dans cet exercice d'ekphrasis continuée, se joue précisément, par-delà la contamination par la peinture et l'enluminure de la tradition allégorique et métaphorique écrite ${ }^{47}$, ce qui distingue l'image verbale de l'image visuelle: la mise en abyme de la création poétique en quoi consiste ce poème allégorique - une vision onirique - réside dans l'affirmation de la nécessité pour l'image peinte d'être prolongée par le langage de la poésie. Je ne m'attarde pas: un article de Rainer Stillers fait excellemment le point sur cette question de l'allégorie quand elle se fait "poétologique $^{48}$ ». La reconnaissance grâce à l'image sensible d'une image mémo-

Natura parte di Sé somigliante / non occultò nell'atto [rédaction B: "nell'arte"] in che suggella". L'image achiropoï̀te a trouvé pour la première fois sans doute une main susceptible de la concurrencer... Il resterait à confronter minutieusement les peintures allégoriques de Boccace tant avec la tradition écrite antérieure (Alain de Lille, de nouveau, pour le palais de Nature dans l'Anticlaudianus [I, 113 sq.], Guillaume de Lorris, L'intelligenza, etc.; v. à propos du dernier texte cité Marcello CICCUTO, "Codici figurati romanzi al servizio di Madonna Intelligenza» in IDEM, Icone della parola. Immagine e scrittura nella letteratura delle origini, Modène, Mucchi, 1995, p. 53 93), qu'avec les reliefs purgatoriaux de Dante et la décoration du palais de Syphax dans l'Africa (III, 87-266) - sans oublier les (modestes) essais précédents de Boccace lui-même dans le Filocolo (II, 32, 1-5 et IV, 85, 2-10).

45. Cf. Amorosa visione, XV, 46-73.

46. À titre d'exemple des épisodes faits pour exciter l'imagination: lorsque Zeus transformé en cygne se fut approché de Léda, celle-ci «In braccia stretto se l'avea pigliato [...] e in camera soletta via portato " (XVII, 79-81). N'oublions pas qu'il s'agit toujours de décrire une peinture!

47. L. BatTAglia RicCI, Boccaccio, cit., p. 109-110.

48. Rainer STILlers, "L'Amorosa visione e la poetica della visualità», in Autori e lettori di 
rielle préexistante, ayant du reste ses origines dans la littérature, met en branle la faculté de produire en un procès virtuellement indéfini des images intérieures qui trouvent alors leur expression accomplie dans le geste discursif, temporalisé, de la poésie. Il n'en reste pas moins que c'est aussi son pouvoir générateur, dans sa densité spécifique, qui est ainsi exalté. L'interexpressivité trouve, dans un tel cadre rhétorico-poétique, à la fois son extension maximale et ses limites indépassables. Au paradigme de la "visibilité» (extérieure) se substitue celui de la "visualité» (intérieure), par où vont se frayer désormais de nouveaux sentiers, le long desquels vont apparaître des images-textes d'un type inédit.

Stillers met en relation la «leçon» de l'Amorosa visione dans sa défense des images avec les plaidoiries en faveur des fictions poétiques figuratives dans les digressions ou développements théoriques sur la poésie des textes postérieurs (Trattatello, Genealogie $)^{49}$. Mais c'est passer par-dessus une autre destinée possible de l'image, enjamber l'une de ses potentialités, dans une direction non prévue par le protocole allégorique - celle qui se déduit du Décaméron. Boccace y parvient par le biais d'une dernière expérimentation, où l'on retrouve les nymphes et dont je voudrais dire à présent quelques mots. Dans le Ninfale fiesolano, après avoir présenté Diane sous les traits topiques $\mathrm{du}$ "canon bref» - mais en ne le recouvrant que "d'un zendado [...] ch'a pena coprire,/sì sottil era, le carni potea ${ }^{50}$ »-, le narrateur introduit la scène, semblable à celle de l'Ameto, où Africo surprend auprès d'une fontaine le groupe des nymphes de la déesse et s'éprend de l'une d'elle. Mais il restreint encore la descriptio, réduite à deux ou trois touches essentielles contenues dans les limites d'un huitain (30), pour s'attarder sur les effets psychologiques produits chez le jeune homme par la rencontre. Jusqu'au moment où il se confie à son père en évoquant la

Boccaccio, Atti del Convegno internazionale di Certaldo (20-22 settembre 2001), éd. M. Picone, Florence, Cesati, 2002, p. 327-342. On mesure ici la distance qui sépare la mise en scène de l'Amorosa visione des passages du Filocolo où les "figures", loin d'être autosuffisantes, sont accompagnées de didascalies qui en éclairent la «signification» (cf. notamment IV 85, 4: «In questa sala ne'pareti dintorno, quante antiche storie possono alle presenti memorie ricordare, tutte con sottilissimi intagli adorne d'oro e di pietre vi vedresti, e sopra tutte scritto di sopra quello che le figure di sotto vogliono significare»).

49. Ibid., p. 341-342.

50. Ninfale fiesolano, 12, 4-5. Mais v. aussi la deuxième rencontre d'Africo avec un groupe de trois nymphes aux huitains 58sq., lorsqu'il aperçoit leurs jambes parce qu'il surprend leurs ablutions ou que celles-ci en s'enfuyant retroussent leurs jupons (situation, cette dernière, qui se reproduit plus loin avec Mensola, 109). 
cause de ses tourments à travers un mensonge transparent: il subit, lui ditil en effet, la fascination d'une jeune biche se dérobant à lui en une poursuite sans trêve. On connaît la suite: Africo, ayant réussi enfin à l'approcher sous de faux-semblants (il se travestit lui-même en nymphe, c'est-à-dire en une image trompeuse, inversée) $)^{51}$, finira par la (i.e. Mensola) posséder, mais au prix de sa vie. L'histoire d'ailleurs bégaie: l'aïeul d'Africo avait déjà connu le même sort et cela aurait dû servir à ce dernier d' "esempletto" (86-92 et rubrique correspondante). L'objet de l'amour, présent dans le cœur de l'amant ${ }^{52}$, a tout l'aspect d'une vision (comme c'est en rêve que, conformément au canon allégorique, Vénus apparait dans le but de seconder le protagoniste); il y a toujours une fontaine ou un fleuve à proximité, attribut indispensable des nymphes et miroir virtuel des scènes évoquées, etc. Et la consommation charnelle, qui advient ici ${ }^{33}$ - évoquée en termes imagés, mais ceux des métaphores obscènes qui connaîtront un peu plus tard les développements que l'on sait -, n'est jamais qu'un viol, acmé de la transgression de la loi imposée par Diane $^{54}$ : c'est toujours la même histoire qui se répète, celle dont nous entretenait Agamben, métaphorisée par le concours de tir au "segno" (rappelons-nous la flèche de Vénus dans le finale du Roman de la Rose) qui voit s'opposer Africo déguisé en nymphe et les nymphes elles-mêmes ${ }^{55}$; l'histoire de l'impossible possession de l'image désirée, de la vaine poursuite du fantasme engendré par l'amour, de la fascination mortelle,

51. Voir notamment le détail de la "vitalba", avec une tige de laquelle Africo se ceint par-dessus son vêtement féminin (Ninfale 210), comme les nymphes de la Comedia delle ninfe, XXVI, 17.

52. A vrai dire, le Ninfale reste allusif quant au topos du visage peint dans le cour de l'amant (cf. les mots d'Africo en 184, 7-8, où il évoque " una ch'io vi vidi tanto bella, / che sempre m'è stata nel cor quella»). Et, sous réserve d'une enquête approfondie, il semble bien qu'hormis quelques occurrences juvéniles (cf. Troiolo à Criseida in Filostrato 8, 15: «Del tutto veggio che m’hai discacciato / del petto tuo, ed io oltre moia voglia / nel mio ancora tengo effigiato / il tuo bel viso con noiosa doglia»; Florio à Biancifiore in Filocolo III 18, 4: "Quante lagrime hanno bagnato il dolente petto, nel quale io continuamente effigiata ti porto, come tu se'!»; mais pas, sauf erreur, dans les Rime d'attribution certaine), Boccace ne soit guère sensible au vieux topos. Si cette remarque trouvait confirmation, il resterait à interpréter une telle indifférence...

53. Elle était, on s'en souvient, interrompue par le réveil (provoqué par l'intensité du désir sur le point d'être satisfait) dans l'Amorosa visione. On voit en tout cas à quelle distance nous sommes aussi bien des enlacements raffinés de la tradition courtoise que, surtout, des consommations allégorisées sur le modèle du Roman de la Rose - où c'est avec une image que, dans un paroxysme d'amphibologie grivoise, le protagoniste fait l'amour et "cueille la rose" (v. 21350sq. dans l'éd. suivante: Guillaume de Lorris et Jean de Meun, Le Roman de la Rose, édition d'après les manuscrits BN 12786 et BN 378, traduction, présentation et notes par A. Strubel, Paris, Librairie Générale Française, [Le Livre de Poche, «Lettres gothiques»], 1992).

54. On remarquera, au titre des procédés «canterini», l'insistance pesante sur l'interdit fait aux nymphes de fréquenter les hommes.

55. Ninfale, 219-223. 
pareille à la mélancolie, dont les yeux sont la cause ${ }^{56}$. La liquéfaction de l'amant "chasseur» de proies qui se dérobent lorsqu'elles ne sont plus les habituelles "fiere ${ }^{57}$ " (et à laquelle fera écho plus tard celle de la nymphe) est une autre version du baiser désespéré de Pygmalion à sa statue ou d'Apollon au laurier ${ }^{58}$.

On le voit: le rapport entre image (sous les espèces de la descriptio ekphrastique) et narration se modifie encore sensiblement dans le Ninfale: l'histoire contée - et ses plis tragiques - procède ici de la transgression continuée de l'interdit attaché à l'image, cette loi qui lui impose de rester telle, protégée par son statut d'objet mental confiné dans une pure intériorité. Le canon réduit à l'essentiel n'a au contraire pour fonction ici que de susciter la mécanique du désir peccamineux : ce dernier ne reste plus contenu dans les limites de l'image (tout en excès fût-elle, dans la Comedia notamment, par rapport à elle-même, c'est-à-dire au type dont elle dérivait); il s'en échappe aussitôt pour lancer et relancer l'enchaînement rapide et inarrêtable des séquences du récit. En termes métapoétiques, on pourrait dire que le crime d'Africo et de Mensola (suivi du suicide du premier et de la punition infligée par Diane à la seconde, qui répondent implacablement à la victoire de Vénus) est d'avoir enfreint les règles en vigueur de la «logique» de l'image poétique. Et l'on remarquera pour finir que la victoire fort ambiguë de Vénus est parachevée malgré tout par la naissance d'un enfant à la destinée «historique» - derrière les traits duquel Mensola désespérée, avant de mourir à son tour, avait recherché l'image de l'amant

56. Voir par exemple 156-168 (et la relative «rubrique»). Voir aussi, pour l'attitude typique du mélancolique, la scène où Africo se mire dans une fontaine, «con la man la gota sostenendo / in sul ginocchio il gomito posa[ndo]»(170-172); il n'y est plus que l'ombre de lui-même (177; Armando Balduino, le commentateur du texte dans l'éd. Tutte le opere..., cit., III, Amorosa visione. Ninfale fiesolano. Vita di Dante, Milan, Mondadori, 1974, paraphrase en note "ombra" par " immagine»; cf. la reprise du texte en éd. de poche ["Oscar Mondadori»], 1997, p. 206).

57. Mais déjà la Caccia di Diana anticipait ce motif: la poursuite à travers bois, éreintante et sans trêve, des bêtes sauvages s'apaise dans la décision des nymphes d'abandonner le service de Diane pour celui de Vénus (qui apparaît sous forme d' "ignuda giovinetta», XVII, 31), préparant la métamorphose des animaux offerts en sacrifice en hommes en chair et en os - le narrateur étant pour sa part « di cervio mutato in creatura / umana e razionale», " uom ritorn[ato] / di brutta belva » (XVIII, 11-12 et 23-24).

58. Voir l'Amorosa visione, XIX, 51 (rédaction B); et cf. déjà par exemple l'Ovide moralizé, pour lequel je renvoie à l'éd. suivante: Ovide moralisé. Poème du commencement du quatorzième siècle, publié d'après tous les manuscrits connus par C. De Boer, Amsterdam, Johannes Müller, I, 1915, v. 3036-3041, p. 126; et, pour Pygmalion et les citations du Roman de la Rose, voir G. Agamben, Stanze, cit., p. 110-111. 
perdu ${ }^{59}$. La levée du voile (de l'«intégument») découvre, non pas une vérité morale cachée, mais, outre la nudité des nymphes (situation récurrente depuis la Caccia di Diana), la vue du désir masculin - et la force de celui-ci convainc la nymphe de donner une suite, pour ponctuelle qu'elle reste, à la première étreinte ${ }^{60}$. L'image "paradisiaque", "angélique», se dissout, y compris sous le signe de la discrète parodisation démythifiante qui parcourt l'œuvre, au profit de la concupiscence charnelle (autrement dit de la «nature» ${ }^{61}$, puis, sur un autre registre, de l'inscription du fruit des amours coupables dans le « réel historique ${ }^{62}$ ».

Le rappel opéré plus haut du récit "mensonger», d'ascendance médiévale rigoureusement littéraire, fait par Africo à son père de sa chasse à la «cerbietta» (76sq.) n'avait pas pour but de nous ramener une nouvelle fois au Politien des Stanze, par exemple, ni même à la réapparition de la biche dans la nouvelle IV 6 du Décaméron (qui nous permettrait pourtant d'ouvrir un autre chapitre sur l'imagination onirique). Mais je souhaiterais à présent le faire entrer en résonance avec la fameuse nouvelle «inachevée», la cent-unième, du Décaméron. Dans le Ninfale, Girafone, qui feint de croire au propos de son fils, lui enjoint de se détourner des «biches»-autrement dit des nymphes - sous peine d'encourir les foudres de Diane, ennemie du genre humain. L'autre père, Filippo Balducci, voulant éviter lui aussi de recourir au «nom propre» des femmes de peur de réveiller l'appétit charnel (le "concupiscibile appetito») de son fils jusqu'alors soigneusement tenu à l'écart d'une telle source de perdition (Africo n'a lui non plus jamais vu de nymphes auparavant, pas plus d'ailleurs que Mensola n'a vu d'homme) ${ }^{63}$, les désigne, on s'en souvient, comme "papere». La dégradation nominaliste du signe ainsi opérée étant motivée, sur le plan interne de la nouvelle «réécrite» par Boccace ${ }^{64}$, par

59. Cf. Ninfale, 405.

60. Cf. ibid. la scène capitale des strophes 235 sq. Pour la reprise apaisée des enlacements amoureux, voir les strophes 309 sq.

61. Ibid., 309.

62. Voir les strophes $11,111,273$, où nous rencontrons, pour les deux premières, la série de rimes «viso ", "paradiso » et " $\operatorname{mir}[\mathrm{ar}]$ fiso »; la troisième commence par une variante qui introduit sur le mode du pressentiment, au moment où vont s'unir Africo et Mensola, le motif de la scissio («I' non potre' giammai stare diviso/da te, dolce mio bene»). L'épithète "angélique» (cf. Ninfale $56,295)$, qui rattache en quelque sorte les nymphes à la poésie amoureuse des origines, était récurrente dans la Comedia delle ninfe (v. II 45, V 1, XI 23, etc.).

63. Cf. Ninfale 269.

64. Cf. la longue tradition qui conflue dans la nouvelle XIV du Novellino: voir à ce propos la note de V. Branca dans l'éd. suivante (à laquelle renverrons les citations ultérieures du texte): 
l'inversion des rôles dans l'acte de nomination (c'est le père qui ici, contrairement à ce qui se passe dans le Ninfale, rebaptise). Le contexte de cette unique nouvelle dont l'auteur du Décaméron s'attribue la responsabilité (mais on vient de rappeler qu'elle n'est pas «originale», elle non plus) la rend, comme l'on sait, plus métapoétique qu'aucune autre. Or, comment la «nature», force de loin supérieure aux artifices d'un père qui avait cru pouvoir la vaincre, s'est-elle frayé la voie de la reconnaissance, quand ni les palais, ni les églises, pas davantage vus précédemment par le jeune homme, ne suscitent chez lui qu'indifférence? Boccace nous suggère que la médiation pourrait avoir été celle de l'image: les jeunes femmes qu'il voit pour la première fois sont encore plus belles que les «anges peints" que son père lui a si souvent montrés sur les parois des églises. Elles correspondent, en mieux, au "déjà vu» de sa mémoire fantasmatique. Autrement dit, la beauté désirable pourrait caractériser d'abord, pour nous qui sommes un composé d'âme et de corps, ces créatures intermédiaires entre monde sensible et monde supra-sensible que sont les anges. Mais que ces derniers soient une sorte d'avatar plus libre, moins soumis aux impératifs de la stylisation, de la figure «nymphéale», que les jeunes Florentines conservent, à côté de leur ressemblance angélique, quelque trait les apparentant aux créatures désirables des textes précédents, c'est ce que pourrait signifier un manuscrit du Décaméron orné de dessins très vraisemblablement dus à la main de Boccace et où l'on voit, dans la troisième «vignette» du dessin illustrant la nouvelle inachevée, six jeunes filles avançant deux par deux ${ }^{65}$. Mais on comprend la leçon: "e vadinsi le ninfe a ripor tutte/ché certo allato a quest [e] sarien brutte», comme l'on pourrait dire en pastichant $\mathrm{Pulci}^{66}$; place donc aux femmes...

La première image, celle des biches du Ninfale, procède d'une construction allégorique autorisée par la tradition; celle du Décaméron correspond au réinvestissement par Boccace de ce qu'il pouvait voir sur les murs des lieux de culte familiers, florentins ou, déjà, napolitains - et l'on pense aux anges de Giotto, toujours évoqué dès qu'il est question des rapports entre Boccace et l'art, mais aussi peut-être d'un peintre que l'on s'étonne un peu

Decameron, nuova edizione rivista e aggiornata, a cura di V. Branca, Turin, Einaudi, 1980, 1992 I, p. 462-463.

65. Il s'agit du ms It. 482 de la $B N F$, copie du Décaméron réalisée par le marchand florentin Giovanni d'Agnolo Capponi dans les années soixante du Trecento. Pour une description de ce manuscrit, cf. la "fiche» $\mathrm{n}^{\circ} 7$ dans Boccaccio visualizzato, cit., II, p. 66-72.

66. Luigi PUlCI, Morgante, XV 101, 7-8. 
de ne pas voir cité dans les études sur ce sujet, et même si Boccace luimême ne le mentionne jamais - je veux parler de Pietro Cavallini, actif à Naples à plusieurs reprises depuis le début du Trecento ${ }^{67}$.

Il y a là quelque chose de quasi blasphématoire, en tout cas de délibérément parodique. Et la parodie devient totale et violente avec la nouvelle IV $2^{68}$. En effet, "donna zucca al vento», qui, selon l'opinion qu'elle a d'elle-même, serait belle même au Paradis ( $\$ 13,20,43)$, croit d'autant plus volontiers à la prétendue vision de «frate Alberto " (quand celui-ci lui raconte qu'il a été visité par l'ange Gabriel et se sert de son invention comme prétexte pour vanter à son tour les «beautés célestes » de celle qu'il convoite $[\$ 18]$ ), et elle accepte d'autant plus vite sa visite nocturne «en forme d'homme", qu'elle a une dévotion toute particulière pour l'ange de l'Annonciation, réveillée par chaque peinture où elle le voit représenté au point d'être jalouse de la Vierge Marie aux pieds de qui il est habituellement agenouillé. Frère Alberto va pouvoir dès lors lui faire connaître la "gloire céleste» $(\$ 32,34)$. En d'autres termes, l'image peinte, son inscription dans la mémoire, a un tel pouvoir de suggestion sur la «vertu imaginative » qu'elle est prête à déborder de son cadre, à remettre en cause son statut d'imago pour s'incarner. Telles sont les puissances de la figure, au sens que donnait André Chastel à ce terme ${ }^{69}$. Mais, à vrai dire, une telle prédisposition à la corporéité ${ }^{70}$ est bien dans sa double nature, abstraite et concrète à la fois: l'image, sous l'impulsion de la peinture telle que la conçoit Boccace dans le sillage de la révolution en cours dans les arts visuels, tourne le dos à l'idolâtrie qui était encore la marque distinctive de l'admiration d'Ameto pour les nymphes, mais de manière paradoxale, c'est-à-dire en la faisant pencher résolument vers le sensible, en la «rematérialisant", en jouant l'imagination corporelle contre l'"alta fantasia", celle qui regarde «intus et sursum ${ }^{71}$ »; s'engouffrant dans la brèche ouverte entre la logique spéculative des clercs et celle, implicite, des œuvres ${ }^{72}$, il restaure, faisant fonds sur l'affectio imaginaria, les droits de la chair, fût-ce

67. S'il ne reste pas grand-chose des fresques de Cavallini dans la cité parthénopéenne, sauf à San Domenico Maggiore (où quelques anges, modestes, accompagnent les histoires des saints qui y sont représentées), on peut facilement imaginer qu'il y avait, parmi les œuvres perdues, l'équivalent de celles du réfectoire de Santa Cecilia à Rome et de leurs éblouissantes théories angéliques.

68. On pourrait y ajouter les reliques - ces autres «images» - de VI 10, où c'est encore l'ange Gabriel qui est convoqué par frate Cipolla.

69. Cf. André CHASTEL, Fables, formes, figures, 2 vol., Paris, Flammarion, 1978, I, p. 38 sq.

70. Rappelons que le titre de l'ouvrage de J.-C. Schmitt déjà cité est précisément Le Corps des images.

71. Cf. E. De Bruyne, Études..., cit., I, p. 598.

72. Cf. J.-C. SCHмitT, Le Corps..., cit., p. 152 et passim. 
au prix d'une attitude totalement irrévérencieuse à l'endroit des images pieuses. Les Victorins nous mettaient en garde: l'imagination est animale, «bestialis», dès lors qu'elle n'est plus gouvernée par la raison, qu'elle cesse d'être «moderatrix ${ }^{73} »$. C'est ce que signifie, je crois, la fin de la nouvelle que je viens d'examiner: l'ange, qui a perdu ses ailes, s'est mis à voler à l'envers, ou plutôt a chuté du ciel vers la terre $(\$ 45,47,55)^{74}$. Puis, pardelà la réminiscence chez Boccace de spectacles de rue, le travestissement en ours (un ours qui semble échappé aussi des marginalia de quelque codex destiné, en sa partie centrale "sérieuse», à la dévotion, qui serait sorti de ces marges où se donnent libre cours toutes les métamorphoses et où s'exprime librement la fantasia des décorateurs ${ }^{75}$ ), le nouveau déguisement du frère dépouillé de son apparence angélique, qui en fait une variante de l'«homme sauvage» (\$54), pourrait être motivé par un effet de symétrie: de part et d'autre de l'homme, en effet, les créatures les plus proches de lui sont l'ange, d'un côté et, du côté animal, l'ours, "roi déchu» des animaux. Celui-ci, selon une longue tradition médiévale, se prête d'autant mieux à la mise en relief de ce qu'il peut y avoir de «bestial » (voire de diabolique) chez l'homme que, dans l'organisation du monde animal, il est perçu comme la créature la plus proche de lui, notamment en ce qui concerne les appétits sexuels ${ }^{76}$. Le renversement de situation est signifié de plusieurs façons: les plumes de l'ange se transmuent en «penna matta", révélant de la sorte leur véritable nature; jusqu’à la relation habi-

73. Cf. E. De Bruyne, Études..., cit., I, p. 593 et n. 3.

74. On notera que dans la tradition de l'illustration de cette nouvelle dans les manuscrits enluminés, c'est à ses ailes qu'on reconnaît le personnage. «[...] io non ho penne / a poter su volar », dit le narrateur à son guide dans l'Amorosa visione (III, 72-73), pour justifier son choix de la voie la plus facile, celle qui conduit aux «ben fallaci» (v. 38) représentés par les peintures des salles du " noble château ", et risquer de se laisser éblouir par le "falso immaginar" que fait naître le "bestial disio" (v. 77 et 88). Dans le De casibus encore (VI, I, 9), en ce qui semble être une autre allusion à Dante, Boccace répond à Fortune qu'il ne possède pas d'ailes lui permettant de pénétrer dans les cieux et d'en explorer les arcanes («Agnosco quidem non esse pennas volucres michi, quarum adiutus suffragio celos penetrare queam, ibidem Dei lustraturus archana...»); seules les "humanae res» lui sont accessibles (ibid., 11).

75. Voir à ce sujet Jean-Claude SCHMITT, «L'univers des marges", in Le Moyen Âge en lumière, sous la direction de J. Dalarun, Paris, Fayard, 2002, p. 329-361.

76. Pour une mise en perspective historique, voir la grande synthèse de Michel PASTOUREAU, L'Ours. Histoire d'un roi déchu, Paris, Seuil, 2007 (cf. en particulier les chapitres «Le parent de l'homme», p. 87-119; «L'ours chez le Diable», p. 153-180; et, pour l'«homme sauvage» au XIV siècle, p. 271-273). C'est en "uomo salvatico" que les illustrateurs prendront l'habitude de représenter frère Alberto (cf. par exemple, encore autour de 1430 à Florence, le ms It. 63 de la $B N F$, f. $138 r$; et en France, quelques années auparavant - entre 1414 et 1419 -, le célèbre ms Pal. lat. 1989, f. $124 v$, dont les illustrations de l'ensemble des cent nouvelles sont dues pour une large part au Maître de la Cité des dames de la Vaticane: pour ce dernier manuscrit, voir Boccaccio visualizzato, cit., III, p. 205-214). 
tuelle entre l'ours et le miel s'en trouve inversée (\$52). L'on se souviendra alors également que c'est en ourse qu'à l'instar de la Callisto d'Ovide Mensola craint d'être transformée par Diane ${ }^{77}$. Ainsi rencontre-t-on une fois de plus chez Boccace, autour d'un même motif, capable en l'occurrence de "faire image», les couches multiples d'un savoir à la fois encyclopédique et étonnamment éclectique, tendant à se recombiner dans une direction où les valeurs morales symboliquement mises en scène ne relèvent plus des protocoles allégoriques.

Pour en revenir, en guise de conclusion, à la nouvelle surnuméraire de l'introduction à la quatrième journée du Décaméron, je voudrais avancer une hypothèse quant à son "inachèvement ": elle pourrait bien n'être telle que parce qu'elle prend fin avant qu'il n'y ait en acte réhabilitation de l'amour humain sous l'égide de la «raison pratique» (au sens boccacien ce qui est l'une des affaires essentielles, sinon la principale, de son grandœuvre). Arrêté sur les "papere» - c'est-à-dire ici aussi sur l'animalité, toute nominaliste qu'elle soit, et même si elle concerne un oiseau domestique infiniment moins inquiétant et ambigu -, le récit en reste en quelque sorte au stade des prolégomènes, autrement dit de la retrogradatio ymaginationis - comme dirait Alain de Lille (qui évidemment la condamne $)^{78}$. Boccace en éprouve la nécessité, lui dont l'imagination se délecte de sa capacité à inverser la signification des icônes, le pouvoir de suggestion des signes visuels ${ }^{79}$. Remplaçant la méfiance (à l'égard de l'idolâtrie) par la confiance (pour une iconodulie laïcisée, promesse de plaisirs terrestres), il en promeut les vertus - si l'on me passe l'expression - «catagogiques », autrement dit antiascétiques, antipénitentielles, ce qui ne laisse

77. Cf. Ninfale, 334, 336; et voir, pour la source latine, Ovide, Métamorphoses, II 422sq. (où c'est Junon qui inflige cette punition à la nymphe de Diane, comme d'ailleurs lors d'autres reprises du mythe par Boccace: cf. les renvois à Filocolo, Teseida, Comedia, Amorosa visione, Elegia, Genealogia que donne en note A. Balduino à la p. 232 de l'éd. cit. du Ninfale). Africo lui-même se sent devenir «animal» (Ninfale, 354).

78. Cf. le Sermo de sphaera intelligibili, donné en appendice à l'Anticlaudianus, éd. cit., p. 322335, p. 334 pour la retrogradatio; un peu plus haut (p. 330), Alain écrit: «Per deuationem enim sensus et ymaginationis fit anima pecus».

79. Voir par exemple à ce sujet le bel article de Winfried WeHLE, "Venus magistra vitae: sull'antropologia iconografica del Decameron", in Autori e lettori..., cit., p. 343-361, sur l'inversion des signes vénusiens traditionnels dans la nouvelle perspective anthropologique que Boccace entend promouvoir. Boccace ne cherche pas à guérir de l'amour, mais à montrer les conditions de l'amour heureux et l'on pourrait prolonger l'analyse sur bien d'autres terrains: ainsi, par exemple, à la ronde obsédante des nymphes, se substituent les «carole» des jeunes femmes du récit-cadre du Décaméron... 
bien sûr de frapper dans le contexte où ces pages sont écrites ${ }^{80}$. On dirait presque qu'il y a des images achiropoïètes chez Boccace, mais c'est la Nature, une nature immanentisée, qui les produit...

En même temps, il me paraît important de redire combien une telle opération fondée sur les prestiges de la peinture (dans l'âme comme sur les murs) transforme la fonction de l'image, de la puissance imaginante pour toute la littérature à venir. On a peut-être trop insisté sur le «droit au réalisme» revendiqué par l'auteur de la Conclusion du Décaméron - la légitimation de la métaphore "douteuse», pour évoquer ce qui n’avait jusqu'alors pas droit de cité dans les lettres, y étant fondée à la fois sur le constat de l'amphibologie potentielle (y compris dans les Ecritures) du signe linguistique (et du signe en général) et sur les nouveaux usages de l'image liés à l'essor des arma Christi, sur le nouveau canon présidant à la représentation sexuée des personnages de l'histoire sainte, et sur les procédés imagés qui avaient la faveur des prédicateurs ${ }^{81}$. On a souvent mis en relief aussi la fonction du recours à Giotto pour l'élaboration (indirecte) d'une théorie de la représentation, finalisée à la fois à la quête de l'illusion et à l'affirmation de l'égale liberté du peintre et du poète, liés par une solidarité refondée - ce que disent éloquemment aussi bien la nouvelle VI 5 du Décaméron que plusieurs paragraphes beaucoup plus tardifs des Genealogie ou des Esposizioni sopra la Comedia di Dante ${ }^{82}$.

80. Cf. L. BatTAgLia RiCCI, Ragionare nel giardino..., cit., en particulier les passages où elle critique la grande thèse de Millard Meiss, p. 47 sq. et passim. Idéal ascétique «redimensionné» par Boccace, en une sorte de réinterprétation du cycle du Camposanto de Pise (outre Battaglia Ricci, voir Marcello CiccuTo, Giunte a una lettura esemplare di Buffalmacco nel Camposanto di Pisa, in IDEM, Icone della parola, cit., p. 113-145) : il suffit de voir la place qu'occupe l'ermitage où s'est retiré Filippo Balducci avec son fils dans l'illustration du ms It. 482 de la $B N F$ déjà cité, littéralement serré, " coincé" dans une bande exiguë entre la scène animée de la mort de l'épouse et mère, et celle de la rencontre avec les "papere", qui est celle qui a droit à l'espace le plus ample. Pour la postérité de l'image des canards devenus oies à l'âge moderne (XVIII' siècle), voir Andreina GRISERI, "Di fronte al Decameron. L'età moderna", in Boccaccio visualizzato, cit., I, p. 155-211, ici p. 186187.

81. Cf. Decameron, Conclusione dell'autore, $\$ 5-7,23$. L'argument est repris, sur la base non plus de la prédication, mais des décorations dans les églises - mises ici en parallèle avec les palais laïcs - dans les Genealogie deorum gentilium, XIV, XVIII, 9. Sur les arma Christi et l'imago pietatis, voir Hans Belting, Das Bild und sein Publikum im Mittelalter: Form und Funktion früher Bildtafeln der Passion, Berlin, Gebr. Mann, 1981 (éd. française L'Image et son public au Moyen Âge, Paris, Gérard Monfort, 1998); sur la sexualité dans l'iconographie médiévale, v. J. WIRTH, L'Image médiévale, cit., p. 322sq.

82. Cit. Dec. VI 5, 5-6; Genealogie deorum gentilium, XIV, VI, 7; Esposizioni, XI, 69. 
Mais le fait que Boccace se montre spectateur exceptionnellement attentif de la peinture de son temps ne peut que nous amener à nous interroger sur le rôle que joue chez lui cette attention au visuel - quand il devient affaire d'ingegno - dans le procès même de la représentation, confié à l'œil intérieur dont se nourrit «l'intellect des sages» («lo 'ntelletto de' savi»; Dec., VI 5, 6). Boccace n'avait selon toute vraisemblance pas encore lu le passage des Institutions oratoires de Quintilien sur la phantasia comme condition de l'enargeia (les visiones intérieures d'où procède l'hypotypose l'inlustratio, l'euidentia) ${ }^{83}$; mais à vrai dire, il n'en avait guère besoin. Il semble en tout cas que, conformément à la leçon qui se dégageait déjà de l'Amorosa visione, la plume de l'écrivain ait la faculté de s'approcher de plus près que le pinceau du peintre (tout mû par l'ingegno que soit celuici) de ce qui est le propre de la nature, grâce aux artifices du langage verbal prenant le relais de l'iconique pur ${ }^{84}$. Mais, et c'est ce qu'il importe de relever ici, il s'agit bel et bien de partir de l'image. Tel est le legs de Boccace à la postérité, qui, tout en maintenant in fine le primat de l'écriture, inverse la hiérachie habituelle en cessant de subordonner absolument la production des images à une finalité consignée dans un texte lui préexistant. Il me revient alors à l'esprit - mais je ne suis pas le premier à faire ce rapprochement - ce que, tout près de nous, Italo Calvino dit de la genèse de sa production narrative; j'ajouterai à la citation par Rainer Stillers de la qua-

83. Il s'agit des $\$ 29 s q$. du chap. II (De divisione adfectuum et quomodo mouendi sint) du livre VI des Institutiones oratoriae. Sur la lecture de Quintilien par Boccace (qu'il cite pour la première fois comme modèle d'éloquence - et c'est peut-être symptomatique - dans la nouvelle de frate Cipolla, VI 10, 7), voir Giuseppe Billanovich, Petrarca letterato. I. Lo scrittoio del Petrarca, Rome, Edizioni di Storia e Letteratura, 1947, p. 156-157.

84. Cf. l'évocation d'Hélène de Troie dans les Esposizioni, V, litt., 102-106 (après avoir rappelé comment Zeuxis à Crotone s'y était pris, en mobilisant toutes les ressources de son "ingegno", pour approcher de la beauté parfaite, Boccace poursuit ainsi: «Nel quale artificio forse si poté abattere lo 'ndustrioso maestro alle lineature del viso, al colore e alla statura del corpo: ma come possiam noi credere che il pennello e lo scarpello possano effigiare la letizia degli occhi, la piacevoleza di tutto il viso e l'affabilità e il celeste riso e i movimenti della faccia e la decenzia delle parole e la qualità degli atti? Il che adoperare è solamente oficio della natura. E, per ciò che queste cose erano in lei esquisite, né vedeano i poeti a ciò poter bastare la penna loro, la finsero figliuola di Giove, acciò che per questa divinità ne desser cagione di meditare qual dovesse essere il fulgore degli occhi suoi, quale il candore del mirabile viso, quanta e quale la volatile e aurea coma [...], quanta fosse la soavità della dolce e sonora voce, e ancora certi atti della bocca vermiglia e della splendida fronte e della gola d'avorio e le delizie del virginal petto, con le altre parti nascose da'vestimenti». Et nous y revoilà, malgré l'âge et les scrupules! L'invention poétique est toujours ici au service de la représentation et de ses pouvoirs sur l'imagination du lecteur. Je cite d'après la reprise en édition de poche Mondadori ["Oscar classici»], Milan, 1994, p. 305-306, du texte de Tutte le opere..., cit., VI: Esposizioni sopra la Comedia di Dante, éd. G. Padoan, Milan, Mondadori, 1965). 
trième "leçon américaine ${ }^{85}$ ", la postface de 1960 (déjà) à l'édition en un volume de la trilogie I nostri Antenati; et j'avance cette suggestion d'autant plus volontiers que l'auteur lui-même y fait brièvement le point sur la légitimité ou non d'une lecture «allégorique» - une telle lecture serait fautive - des trois récits ainsi commentés ${ }^{86}$. In principio erat imago...

Les choses s'articulent de manière certes beaucoup plus complexe que cela chez Boccace (comme du reste chez Calvino), même dans les cas où, comme en IV 2, on peut soupçonner que l'image a joué un rôle moteur initial; il n'en reste pas moins qu'elle est très souvent en elle-même génératrice d'action, et que le récit de cette dernière non seulement appelle, suscite de nouvelles images, mais agit en retour sur l'image première. C'est ce que je voudrais illustrer rapidement à présent. La mise en abyme du regard est elle-même, dans certains cas remarquables, le produit d'une image. Un exemple va nous permettre d'aller un peu plus loin dans l'analyse.

Il s'agit de la célèbre nouvelle V 1 du Décaméron, destinée selon son narrateur (Panfilo le bien nommé) à montrer combien les forces de l'amour sont puissantes et bénéfiques. On se rappelle l'épisode initial: le stupide Cimone, relégué par son père à la campagne, tombe un jour, en se promenant à midi dans une clairière, sur la belle Efigenia endormie près d'une fontaine. L'esquisse très rapide et suggestive des charmes à peine voilés de la jeune femme a un effet immédiat: Cimone, comme fasciné, s'appuie sur son bâton et se met à la contempler. Son hébétude, bien conforme à sa nature, est cependant vite ébranlée et il devient étonnamment capable, après en avoir "distingué les parties" en un nouvel exercice ekphrastique

85. R. STILLERS, «L'Amorosa visione..., cit., p. 327.

86. Cf. I nostri antenati. Il visconte dimezzato, Il barone rampante, Il cavaliere inesistente, Turin, Einaudi, $1960^{12}$, p. 354. Après avoir affirmé qu'il n’a jamais eu "alcuna intenzione d'allegoria moralistica o, meno che mai, politica in senso stretto", Calvino précise ceci : "All'origine di ogni storia che ho scritto c'è un'immagine che mi gira per la testa, nata chissà come e che mi porto dietro magari per anni. A poco a poco mi viene da sviluppare questa immagine in una storia con un principio e una fine, e nello stesso tempo - ma i due processi sono spesso paralleli e indipendenti - mi convinco che essa racchiude qualche significato ". Il revient de façon plus développée et articulée sur ce point dans les Lezioni americane, Milan, Garzanti, 1988, p. 88 sq. ; après avoir rappelé que "possiamo distinguere due tipi di processi immaginativi : quello che parte dalla parola e arriva all'immagine visiva e quello che parte dall'immagine visiva e arriva all'espressione verbale» (p. 83), et que la modernité «inclin[a] decisamente dalla parte dell'immagine visuale» (p. 86). En outre, quand Calvino cite Boccace dans ces mêmes «leçons" pour faire l'éloge de la «légèreté» (à propos de VI 9, la nouvelle de Guido Cavalcanti), il convient de remarquer que la scène, au milieu des «avelli» de Santa Maria Novella, est à sa manière puissamment visuelle (p. 12-13). 
de descriptio puellae (complété cette fois par le nez!), d'en reconnaître le caractère quasi-divin. On sait la suite: l'image commence à mettre en mouvement chez lui la raison, seulement endormie jusque-là; le réveil d'Efigenia, dont il va enfin être en mesure de voir les yeux, va finir de briser le sortilège: réagissant à la fixité du regard du jeune homme, la jeune fille s'éloigne, ce qui a pour effet de provoquer le miracle des progrès fulgurants du «bestione», du «montone» (de l'«insensato animale», \$ 56) vers son humanité pleinement recouvrée. On a reconnu au passage tous les éléments topiques, savamment recomposés, de la tradition lyrique antérieure, jusqu'à l'hyperbolisation des vertus ennoblissantes de l'amour. Tout cela n'étant que prétexte à la mise en route de la machine narrative, à l'enchaînement rapide de ses péripéties jusqu'à l'enlèvement des deux jeunes femmes et au dénouement heureux du double mariage.

Mais ce qui m'intéresse au premier chef ici, c'est l'attitude de Cimone telle qu'elle est d'abord saisie. Pour le lecteur de Boccace, elle est familière: que l'on se reporte notamment au début du chapitre V (\$ 1-3) de la Comedia delle ninfe ${ }^{87}$. La sidération qui frappe d'abord ces personnages boccaciens au moment de la rencontre avec l'amour en fait pour quelques instants des représentants de l'amor hereos, de la pathologie en quoi consiste celui-ci; ils présentent en effet des symptômes proches de ceux de l'affection mélancolique, des effets dévastateurs du «démon de midi» (et c'est sur le coup de midi que Cimone rencontre Efigenia) et de l'acedia, voire de la vision nocturne inspirée par le diable ${ }^{88}$. L'immobilité médusée, les mains appuyées sur le bâton et la tête reposant sur celles-ci, d'Ameto et de Cimone suggèrent fortement un tel rapprochement. L'hypothèse serait

87. "Ameto, poi che de' cani gli fuggì la paura e l'angelica voce ebbe ricominciata la bella canzone, con timido passo a quelle [les nymphes] si fece vicino; e poggiato in terra il noderoso bastone, sopra la sommità di quello compose ambo le mani, e sopra esse il barbuto mento fermato, come se quivi non fosse, fiso la cantante, alienato, mirava; la quale, poi che ebbe posta fine alle sue note, dopo lungo spazio, cotale in sé si mosse quale colui che da profondo sonno è a vigilia subito rivocato, il quale, gli occhi volgendo sonnolenti in giro, quasi appena conosce dove sia [...] Egli appena, aiutandolo la forte mazza, in piè rimase, ma pur si sostenne; e poi che tutto fu del preso stordimento uscito, quivi, senza niente parlare a quelle, si pose sopra l'erbe a sedere; e, rimirando la bella ninfa [...]»; suit la description de Lia. On notera ici aussi que toute la scène se déroule depuis le début (chap. III) à l'heure où le soleil est le plus chaud.

88. Voir G. Agamben, Stanze, cit., p. 41-45 et 184-206. Pour la phénoménologie «mélancolique», voir aussi bien sûr (y compris pour les illustrations) Raymond KLIBANSKY, Erwin PANOFSKY et Fitz SAXL, Saturn and Melancholy. Studies of natural Philosophy, Religion and Art, London, Nelson, 1964 (éd. française Saturne et la mélancolie. Etudes historiques et philosophiques: nature, religion, médecine et art, traduit de l'anglais et d'autres langues par F. Durand-Bogaert et L. Evrard, Paris, Gallimard, 1989); ainsi que Mélancolie. Génie et folie en Occident, sous la dir. de J. Clair, catalogue de l'exposition, Galeries nationales du Grand Palais (Paris, 10 octobre 200516 janvier 2006), Paris, Réunion des musées nationaux/Gallimard, 2005. 
à corroborer par une enquête plus vaste et minutieuse. Mais le type «mélancolique» qui se répète à travers les manuscrits enluminés se retrouve, en pleine adéquation avec sa représentation, dans le portrait de ce vrai «malade» qu'est Africo tel qu'il apparaît dans les huitains 170-172 du Ninfale fiesolano - un Africo au désespoir parce que séparé de sa belle, au moment où il se mire dans une fontaine et s'y voit défigurée ${ }^{89}$.

Ce qu'il convient en tout cas de remarquer aussitôt, c'est que Boccace renverse une nouvelle fois le topos. Dans le cœur de Cimone, l'image et son pouvoir de fascination (au sens propre) font place à des "pensées" (leur caractère «discursif» est soigneusement souligné; cf. $\$ 8$ ). Le second commentaire du narrateur (après l'introduction) est explicite (cf. les $\$ 21$ 22) : au lieu de lier les «esprits» du jeune homme, Amour les délie. La "solution" (au sens propre ici encore) va dès lors résider tout entière dans le déploiement de l'action. L'aliénation ${ }^{90}$ de l'amoureux totalement absorbé un temps dans sa contemplation, par la grâce d'un simple regard qui se dérobe, suscitant le désir de l'objet et non de l'image, va l'amener à la maîtrise de soi et de son destin. Le résumé de la nouvelle tel qu'il a été proposé plus haut, ainsi que l'analyse qui vient d'en être faite sont confirmés de façon éclatante, je crois, par le triple dessin à la plume, dû selon toute vraisemblance à Boccace lui-même, qui orne le manuscrit parisien du Trecento déjà cité ${ }^{1}$ et qui constitue une véritable interprétation de la nouvelle - bien davantage qu' une simple illustration. Trois «vignettes» se succèdent (et l'on rappellera que c'est là une grande caractéristique de ces superbes illustrations, parmi les plus résolument narratives de toute la tradition): on y voit d'abord Cimone en «mélancolique», puis on assiste au premier enlèvement en pleine mer "à la pirate»; c'est enfin le second (et dernier) coup de force commis dans le palais des fiancés officiels qui conclut la série. Ce qui aurait risqué, à un stade antérieur, d'être le point de départ d'une nouvelle ronde irritante des nymphes, ou de la quête éperdue, au rythme de huitains se succédant sans relâche, d'une image fuyante comme dans le Ninfale, se résout ici dans le récit des aventures (des "casi») de Cimone, désormais pourvu d'intelligence et capable de parvenir à ses fins, malgré les accidents de fortune dont son parcours est semé.

89. Cf. Ninfale, 172, 1-2: «E con la man la gota sostenendo, / in sul ginocchio il gomito posava [...]. Pour une illustration du XV siècle florentin, v. Boccaccio visualizzato, cit., II, p. 98.

90. On pourrait citer aussi l'aliénation de Filocolo devant le spectacle de Biancifiore qu'il voit nue pour la première fois (cf. Filocolo, IV, 118, 5-6: «Egli, tirate indietro le cortine, con più aperto lume la riguarda e sovente l'anima alienata richiama. »; il s'agit de la phrase qui précède le passage cité à la note 21 ci-dessus).

91. Cf. ms It. 482 de la $B N F$, f. $102 r$. 
Le texte de la nouvelle tout entière rétroagit alors sur le type de l'amoureux sidéré: ce qui aurait pu être une manifestation de plus des dangers de l'amour devient exaltation de son pouvoir libérateur. L'image se transfigure: toute la tradition ultérieure s'en ressentira, qui pourra la combiner positivement avec les virtualités inscrites dans le "canon long" de la descriptio mulieris. Vittore Branca a suivi l'évolution de ce thème iconographique ayant connu une exceptionnelle diffusion, depuis les premières illustrations des manuscrits jusqu'à la Venise du Cinquecento (où il croise et contamine sans doute d'autres sujets), puis à ses métamorphoses ultérieures, baroques et néo-classiques, mais en passant d'abord par le filtre de la (violente) récupération platonisante dans la Florence de Laurent le Magnifique et de Botticelli - où Cimone devient, à côté d'autres figures "saturniennes», l'une des incarnations possibles de l'aspiration à la contemplation ${ }^{92}$. Telle est l'une des conséquences majeures de l'immense pouvoir de séduction qu'exercent les images boccaciennes sur le lecteur: celui-ci est conduit à les prolonger, à les adapter, les modifier, à en produire à son tour de nouvelles, soit dans le for de son sens intérieur, soit dans l'extériorisation de l'imagination quand, comme la nature elle-même dont elle semble tirer son pouvoir, elle se fait pictrix - au sens matériel et concret du terme.

92. Cf. Vittore BRANCA, «Interespressività narrativo-figurativa e rinnovamenti topologici e iconografici discesi dal Decameron", in Boccaccio visualizzato, cit., I, p. 39-74, ici p. 39-50; v. aussi A. GrISERI, «Di fronte al Decameron... », cit., p. 168-178 (pour Cimone et Efigenia). 
